\title{
INCIDÊNCIA E FATORES DE RISCO DE INFECÇÃO DE SÍTIO CIRÚRGICO: REVISÃO INTEGRATIVA
}

\author{
Gabriela do Carmo Santos \\ Universidade Federal de Goiás - Regional Jataí \\ gabrielaenfufg@gmail.com \\ Ana Flávia Guimarães Baylão \\ Universidade Federal de Goiás- Regional Jataí \\ anabbaylao@hotmail.com \\ Samella Cristine Ferreira Borges \\ Universidade Federal de Goiás- Regional Jataí \\ samella.cris@hotmail.com \\ Luiz Almeida da Silva \\ Universidade Federal de Goiás- Regional Jataí \\ enferluiz@yahoo.com.br \\ Mikael Henrique de Jesus Batista \\ Universidade Federal de Goiás- Regional Jataí \\ mikael.gyn@hotmail.com \\ Giulena Rosa Leite \\ Universidade Federal de Goiás - Regional Jataí \\ giulenar@gmail.com
}

\section{RESUMO}

Revisão integrativa da literatura, realizada a partir de fontes secundárias, através de levantamento bibliográfico na base de dados LILACS e biblioteca virtual SciELO com o objetivo de determinar, baseado nas produções científicas publicadas nos últimos 10 anos, a incidência de infecção de sítio cirúrgico e dos fatores de risco relacionados à ela. Foram selecionados para amostra 13 trabalhos, 5 $(38,46 \%)$, apresentaram incidência de infecção de sítio cirúrgico menor que $10 \% ; 7$ (53,84\%) não continham dados sobre os microorganismos mais prevalentes; quanto aos fatores de risco, a prevalência foi de indivíduos do sexo masculino 6 (46,15\%); idade superior a 50 anos $8(61,53 \%)$; cirurgia do aparelho digestivo 4 (30,76\%); não trouxe dados sobre a duração do procedimento cirúrgico 8 (61,53\%); não trouxe dados sobre o tempo de internação prévia $10(76,92 \%)$ e não trouxe dados sobre o tempo de internação total $9(69,23 \%)$. A incidência de infecção de sítio cirúrgico está em processo de redução, mas continua sendo preocupação para os estabelecimentos de saúde; destaca-se a importância de mais pesquisas e trabalhos sobre o tema, visto que através do conhecimento dos fatores de risco, tais infecções tornam-se preveníveis.

Palavras-chave: Infecção da ferida operatória, Infecção hospitalar e Fatores de risco. 
.

v.11, n. 1, 2015

\title{
INCIDENCE AND RISK FACTORS OF SURGICAL WOUND INFECTION: INTEGRATIVE LITERATURE REVIEW
}

\begin{abstract}
A integrative literature review, performed from secondary sources, through a bibliographical survey on database Lilacs and Scielo virtual library, with goal to appoint, based on published scientific productions in last 10 years, the incidence of surgical wound infection and the risk factors related to it. Were selected for sample 13 studies, $5(38,46 \%)$, showed incidence of surgical wound infection less than $10 \%$; 7 (53,84\%) didn't contain data of most prevalent microorganismis; in terms of risk factors, in most studies, the prevalence was males $6(46,15 \%)$; age over than 50 years old 8 $(61,58 \%)$; digestive tract surgery $4(30,76 \%)$; didn't contain data about the duration of surgical procedure $8(61,76 \%)$; didn't contain data about the time of previous internation $10(76,92 \%)$ and didn't contain data about the total time of internation $9(69,23 \%)$. The incidence of surgical wound infection are in reduction process, but remains concern to health establishments; highlights the importance of further research and studies about the subject, as through knowledge of risk factors, such infections become preventable.
\end{abstract}

Keywords: Surgical wound infection, Nosocomial infection, Risk factors.

\section{INTRODUÇÃO}

As infecções hospitalares (IH) configuram-se em graves problemas, crescendo tanto em incidência quanto em gravidade (POVEDA; GALVÃO; HAYASHIDA, 2003). As IH podem ser imputadas à área hospitalar e se manifestar ainda durante o período de internação ou após a alta hospitalar. É visível a sua importância, porque aumentam a morbidade, mortalidade e os custos hospitalares (MARTINS et al, 2008).

O Center for Disease Control (CDC) verificou que o paciente que evolui para uma infecção pode ter seus custos hospitalares triplicados, quando comparado ao paciente que não teve infecção (CDC, 1999).

Em algumas $\mathrm{IH}$, quando se pode intervir na corrente de transmissão dos microrganismos e evitá-las, temos a denominação de infecções evitáveis. A descontinuação dessa corrente pode ser realizada por meio de medidas simples, tais como a lavagem das mãos, o processamento do material 


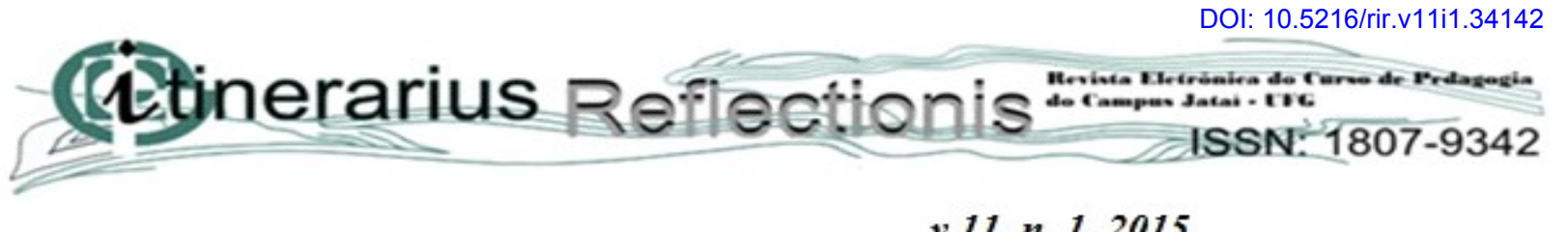

v.11, n. 1,2015

cirúrgico e das superfícies, a utilização dos equipamentos de proteção individual, e o cumprimento das medidas de assepsia (PEREIRA et al, 2005).

Segundo a Agência Nacional de Vigilância Sanitária (ANVISA), as IH ocupam a terceira posição entre todas as infecções em serviços de saúde e compreende de $14 \%$ a $16 \%$ daquelas encontradas em pacientes hospitalizados (BRASIL, 2013).

A infecção que ocorre em pacientes submetidos a procedimentos cirúrgicos no local da operação é definida como infecção de sítio cirúrgico (ISC). No âmbito das infecções hospitalares, o Sítio Cirúrgico tem sido mencionado como um dos principais locais de infecção, contribuem para cerca de $15 \%$ de todas as infecções relacionadas à assistência à saúde, levando a um aumento médio de 60,0\% no tempo de internação (OMS, 2009; OLIVEIRA; CIOSAK, 2007).

Apesar da evolução tecnológica na área cirúrgica e do crescente conhecimento sobre os fatores de risco para as infecções, nas últimas décadas, os índices de ISC ainda são elevados (BURGATTI; LACERDA, 2009).

Na maioria das vezes, a notificação da infecção cirúrgica, é restrita apenas àqueles processos identificados durante a permanência hospitalar e, portanto, não fornece taxas fidedignas, subestimando as verdadeiras taxas, visto que, vários estudos mostram que uma parcela importante de ISC se desenvolve após a alta(OLIVEIRA; CIOSAK, 2007).

As consequências desta subnotificação são muitas, destacando-se a obtenção de taxas irreais que permitem a criação de uma falsa realidade de que não há problemas, e impedem ações que visem melhorias do serviço prestado (OLIVEIRA et al, 2002).

Têm sido relacionados à incidência de ISC fatores intrínsecos (relacionados ao paciente) e fatores extrínsecos (relacionados ao ambiente, membros da equipe, materiais etc). O CDC considera como fatores relativos ao paciente: idade, tipo de cirurgia, doença de base, doenças associadas, entre outros; e como fatores externos os procedimentos assistenciais (técnica cirúrgica, preparo préoperatório, ambiente, paramentação da equipe e outros) (CDC, 1999).

Muitos fatores como a esterilização dos materiais, o número de pessoas na sala cirúrgica e experiência da equipe podem ser responsáveis pelo aumento da taxa de infecção. Tem-se, portanto, que a prevenção e o controle da ISC dependem da adesão dos profissionais às medidas preventivas (CARNEIRO et al, 2013; CUNHA et al, 2011). 
A Associação Paulista de Estudos e Controle de Infecção Hospitalar, baseada no projeto Study on the Efficacy of Nosocomia Infection Control (SENIC), produzido pelo CDC, salienta que a investigação dos fatores relacionados à incidência, morbiletalidade e custos da ISC, podem auxiliar na prevenção de até $20 \%$ dos casos, através da vigilância epidemiológica associada à implementação de programas de controle que, na maioria destes a categoria de enfermagem está inserida (POVEDA; GALVÃO; HAYASHIDA, 2003).

Destaca-se, assim, a importância da equipe de enfermagem no controle e prevenção destas infecções, bem como seu papel crítico como vetor de transmissão, por meio das mãos, durante o contato com os pacientes. O enfermeiro deve, através da educação continuada, repassar conhecimento e informações técnicas à sua equipe e aos outros profissionais envolvidos nestas situações, contribuindo, significativamente, para minimizar a ocorrência das ISC e suas graves consequências (ERCOLE; CHIANCA, 2002).

Diante do exposto, entende-se ser de extrema importância que o enfermeiro compreenda os fatores que predispõem à incidência de ISC, a fim de implementar ações que possibilitem minimizar a ocorrência e os riscos da mesma, contribuindo então, para a melhoria da qualidade da assistência prestada ao paciente; e a fim de possibilitar a implementação do sistema de vigilância, dando aos profissionais do controle de IH o acesso a informações que sirvam de subsídio para a atuação destes, frente às medidas de prevenção e controle das infecções.

Diante dos fatos, e da relevância do tema, este estudo objetivou determinar, baseado nas produções científicas publicadas nos últimos 10 anos, a incidência de infecção do sítio cirúrgico (ISC) e dos fatores de risco relacionados à ela.

Visando o alcance deste objetivo, utilizou-se uma revisão integrativa com base no referencial teórico da prática baseada em evidências. 


\section{MÉTODO}

O presente estudo trata-se de uma revisão integrativa da literatura, realizada a partir de fontes secundárias, por meio de levantamento bibliográfico em material eletrônico.

A revisão integrativa determina o conhecimento atual sobre uma temática específica, já que é conduzida de modo a identificar, analisar e sintetizar resultados de estudos independentes sobre o mesmo assunto, contribuindo, pois, para uma possível repercussão benéfica na qualidade dos cuidados prestados ao paciente (SILVEIRA, 2005).

Para tanto, a presente revisão foi desenvolvida utilizando-se as seis fases propostas por Mendes, Silveira, Galvão (2008):

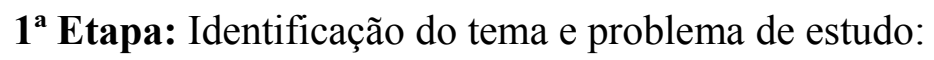

Para buscar o alcance dos objetivos propostos, elegeu-se como questão temática do presente estudo: Quais são os fatores de risco, a incidência e os principais agentes causadores de ISC disponíveis na literatura nos últimos 10 anos?

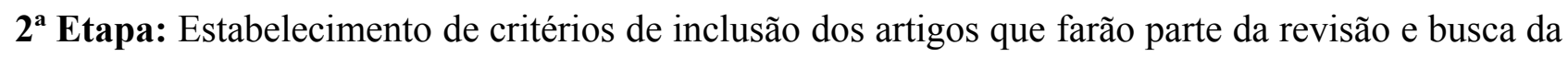
literatura nas bases de dados.

Os artigos foram buscados na base de dados LILACS (Literatura Latino-Americana e do Caribe em Ciências da Saúde) e na biblioteca virtual SciELO Brasil (Scientific Electronic Library Online) sob os descritores: Infecção da ferida operatória, Infecção hospitalar e Fatores de risco.

Foram considerados para esta revisão integrativa apenas estudos primários que levantaram a incidência e epidemiologia das infecções de sítio cirúrgico; artigos de texto completo e em língua portuguesa, publicados a partir do ano de 2003, e que de fato se relacionam com o tema desta revisão.

Foram excluídos, portanto, artigos em língua estrangeira, artigos cujo texto completo não 
estava disponível na modalidade free, estudos secundários, cartas ao leitor, réplicas e duplicatas, editais, opiniões e comentários, análises da literatura, artigos que contemplavam os descritores de assunto, mas que faziam referência a outro tema, e trabalhos publicados anteriormente ao período especificado como critério de inclusão (a partir do ano 2003). A busca de artigos e a elaboração do trabalho foram realizadas no período de novembro de 2013 a janeiro de 2014.

A pesquisa foi realizada por três dos pesquisadores de forma independente; posteriormente, foi feito o cruzamento das três buscas. Foram encontrados 408 artigos indexados nas bases de dados consultadas, sendo: 343 na base LILACS e 65 na Biblioteca Virtual SciELO; após análise criteriosa, definiu-se a amostra em 13 artigos que atenderam aos critérios de inclusão, sendo 06 da base LILACS e 07 da Biblioteca SciELO. As estratégias de busca estão descritas na Quadro 1.

Quadro 1: População e amostra dos artigos indexados nas bases eletrônicas antes e após serem submetidos aos critérios de inclusão.

\begin{tabular}{llll}
\hline Fonte & Estratégia de busca & População & Amostra \\
\hline LILACS & $\begin{array}{l}\text { "Infecção da ferida operatória" or } \\
\text { "Infecção hospitalar" and "Fatores } \\
\text { de risco" }\end{array}$ & 06 \\
\hline SCIELO & $\begin{array}{l}\text { "Infecção da ferida operatória" or } \\
\text { "Infecção hospitalar" or "Fatores de } \\
\text { risco" }\end{array}$ & & \\
\cline { 2 - 4 } & "Infecção da ferida operatória" & 38 & 06 \\
\hline TOTAL & & $\mathbf{4 0 8}$ & $\mathbf{1 3}$ \\
\hline
\end{tabular}

$3^{\text {a }}$ Etapa: Definição das informações que serão extraídas dos estudos selecionados/categorização dos estudos.

Nesta etapa, buscou-se extrair da amostra as informações sobre a incidência e os fatores de risco para ISC relacionados ao microorganismo (patógenos mais frequentes), a incidência dos fatores de risco para ISC relacionados ao paciente (idade e sexo) e sobre a incidência dos fatores de 


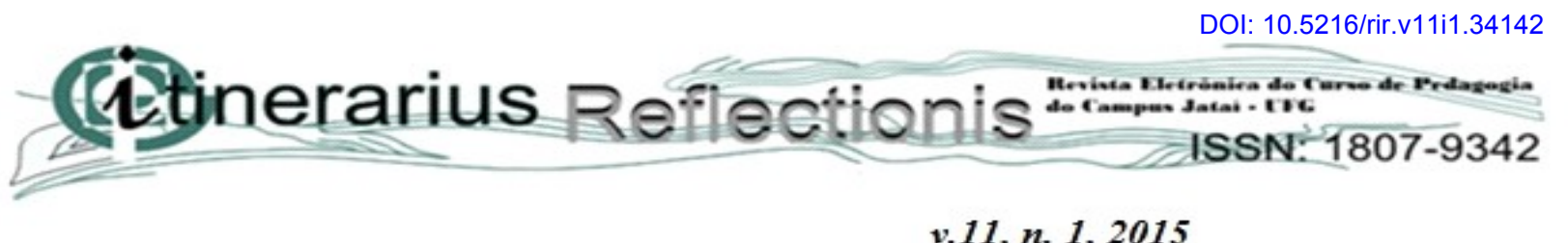

v.11, n. 1, 2015

risco para ISC relacionados ao procedimento cirúrgico (local de cirurgia, tempo de cirurgia, tempo de internação prévio e tempo de internação total).

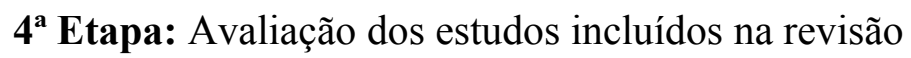

Após extração dos dados, os mesmos foram categorizados e apresentados de forma descritiva, possibilitando o entendimento dos resultados encontrados.

$5^{a}$ Etapa: Interpretação dos resultados

Os dados foram interpretados à luz da literatura científica, comparando os achados entre os autores, identificando pontos de divergência e convergência, discutindo com a realidade do contexto atual, identificando possíveis lacunas do conhecimento, delimitando prioridades para estudos futuros e salientando conclusões e inferências, conforme recomenda Mendes, Silveira, Galvão (2008).

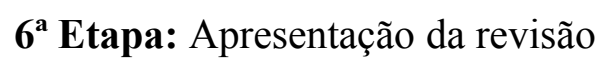

Após a seleção da amostra, os dados encontrados foram tabulados com frequência simples, considerando dois números após a vírgula e em categorias: incidência de ISC, patógenos mais frequentes, idade, sexo, tipo de cirurgia, duração da cirurgia e tempo de internação. Após a tabulação, os dados foram analisados, dando subsídio à discussão.

\section{Resultados}

Vários fatores têm sido relacionados à incidência de ISC, tais como os referentes ao microrganismo, os fatores relacionados ao paciente e os fatores relacionados ao procedimento cirúrgico. Neste trabalho, buscou-se extrair de estudos publicados, dados neste sentido que pudessem subsidiar esta teoria.

A tabela 1 caracteriza os estudos incluídos como amostra nessa revisão integrativa, totalizando 13 estudos contemplativos do tema em questão, sendo organizados em ordem crescente de acordo com o ano de publicação. 
Tabela 1: Descrição dos estudos incluídos na revisão integrativa, segundo autor(es), base de dados e ano de publicação.

\begin{tabular}{|c|c|c|c|c|}
\hline $\mathbf{E}$ & Autor & Título & Ano & Fonte \\
\hline 1 & $\begin{array}{l}\text { FERRAZ, A. A. B.; } \\
\text { FERRAZ, E. M. }\end{array}$ & $\begin{array}{l}\text { Infecção latente de sítio cirúrgico: } \\
\text { hipótese ou realidade? }\end{array}$ & 2003 & SCIELO \\
\hline 2 & $\begin{array}{lll}\text { POVEDA, } & \text { V. } & \text { B.; } \\
\text { GALVÃO, } & \text { C. } & \text { M.; } \\
\text { HAYASHIDA, M. } & \end{array}$ & $\begin{array}{l}\text { Análise dos fatores de risco } \\
\text { relacionados à incidência de } \\
\text { infecção do sítio cirúrgico em } \\
\text { gastrocirurgias. }\end{array}$ & 2003 & LILACS \\
\hline
\end{tabular}

\begin{tabular}{llll}
\hline 3 BARBOSA, H. F.; et al. & Fatores de Risco para Infecções de 2004 & LILACS \\
& Sítio Cirúrgico em Pacientes & \\
& Operadas por Câncer de Mama.
\end{tabular}

\begin{tabular}{lll}
\hline GUTIÉREZ, M. G. R.; & Infecção no sítio cirúrgico: 2004 LILACS \\
et al. & vigilância pós-alta precoce de \\
& pacientes submetidas à cirurgia \\
& oncológica de mama.
\end{tabular}
5 OLIVEIRA, $\quad$ A. $\quad$ C.; Infecção de sítio cirúrgico no $2004 \quad$ SCIELO CIOSAK, S. I. seguimento pós-alta: impacto na incidência e avaliação dos métodos utilizados.

\begin{tabular}{lllllll}
\hline 6 & POVEDA, & V. & B.; & Frequência de infecção do sítio 2005 & SCIELO \\
& GALVÃO, C. M. & & cirúrgico em gastrectomia. & & & \\
& & &
\end{tabular}




\begin{tabular}{lllllll}
\hline 7 & POVEDA, & V. & B.; & Fatores predisponentes à infecção & 2005 & SCIELO \\
& GALVÃO, & C. & M.; & do sítio cirúrgico em gastrectomia. & &
\end{tabular}

SANTOS, C. B.
8 FALAVIGNA, A.; et al. Manejo da infecção após cirurgia 2006 LILACS de fixação interna da coluna lombar.
9 OLIVEIRA, A. C.; Infecção de sítio cirúrgico em 2007 LILACS CIOSAK, S. I. hospital universitário: vigilância pós-alta e fatores de risco.

10 MARTINS, M. A.; et $\begin{aligned} & \text { Vigilância pós-alta das infecções } \\ & \text { al. } \\ & \\ & \text { de sítio cirúrgico em crianças e } \\ & \text { adolescentes em um hospital } \\ & \text { universitário de Belo Horizonte, } \\ & \text { Minas Gerais, Brasil. }\end{aligned}$

11 FALAVIGNA, A.; et al. Evolução clínica e funcional dos 2009 SCIELO pacientes com infecção após artrodese de coluna lombar.

\begin{tabular}{|c|c|c|c|c|}
\hline 12 & $\begin{array}{l}\text { ASSUNÇÃO, T. P.; } \\
\text { PONTES, B. C. D.; } \\
\text { DAMASCENO, C. A. } \\
\text { V. }\end{array}$ & $\begin{array}{l}\text { Prevalência de } \text { infecções em } \\
\text { suturas de cirurgias de } \\
\text { revascularização do miocárdio. }\end{array}$ & 2011 & SCIELO \\
\hline$\overline{13}$ & $\begin{array}{l}\text { SASAKI, V. D. M.; et } \\
\text { al. }\end{array}$ & $\begin{array}{l}\text { Vigilância de infecção de sítio } \\
\text { cirúrgico no pós-alta hospitalar de } \\
\text { cirurgia cardíaca reconstrutora. }\end{array}$ & 2011 & $\overline{\text { SCIELO }}$ \\
\hline
\end{tabular}

No que diz respeito aos patógenos, em mais da metade dos trabalhos, não havia dados sobre os microorganismos mais prevalentes e em três trabalhos $(23,06 \%)$ o microorganismo mais 
v.11, n. 1,2015

frequente foi o Staphyloccocus aureus, conforme apresentado na tabela 3.

Tabela 3: Incidência de infecção do sítio cirúrgico e patógenos mais frequentes conforme amostra.

\begin{tabular}{|c|c|c|c|}
\hline & & $n$ & $\%$ \\
\hline & $<10 \%$ & 5 & 38.46 \\
\hline & Entre $10 \%$ e $20 \%$ & 4 & 30.77 \\
\hline \multirow[t]{3}{*}{ Incidência de ISC } & $>20 \%$ & 3 & 23.07 \\
\hline & Não informado & 1 & 7.70 \\
\hline & Total & 13 & 100 \\
\hline \multicolumn{4}{|c|}{ Patógenos mais } \\
\hline \multirow[t]{5}{*}{ frequentes } & Staphyloccocus aureus & 3 & 23.06 \\
\hline & Pseudomonas aeruginosa & 1 & 7.70 \\
\hline & Escherichia coli & 1 & 7.70 \\
\hline & Klebsiella pneumoniae & 1 & 7.70 \\
\hline & Não informado & 7 & 53.84 \\
\hline & Total & 13 & 100 \\
\hline
\end{tabular}

Quanto ao sexo das vítimas mais acometidas por ISC, a amostra evidenciou prevalência de indivíduos do sexo masculino e com idade maior que 50 anos entre os portadores de ISC. Conforme apresentado na tabela 4 . 
Tabela 4: Incidência de fatores de risco para ISC relacionados ao paciente conforme amostra

\begin{tabular}{clcc}
\hline & & $\boldsymbol{n}$ & $\mathbf{\%}$ \\
\hline \multirow{2}{*}{ Sexo } & Masculino & 6 & 46.15 \\
& Feminino & 3 & 23.08 \\
\hline & Não informado & 4 & 30.77 \\
\hline Idade & Total & $\mathbf{1 3}$ & $\mathbf{1 0 0}$ \\
& $\leq 50$ anos & 1 & 7.70 \\
\cline { 2 - 4 } & $>50$ anos & 8 & 61.53 \\
\cline { 2 - 4 } & Não informado & 4 & 30.77 \\
\hline & Total & $\mathbf{1 3}$ & $\mathbf{1 0 0}$ \\
\hline
\end{tabular}

A respeito do procedimento cirúrgico, dos artigos que trouxeram dados a respeito do local de cirurgia, evidenciou-se que na maior parte da amostra, a prevalência foi das cirurgias realizadas no aparelho digestivo, conforme apresentado na tabela 5 .

Tabela 5: Incidência de fatores de risco relacionados ao procedimento cirúrgico conforme amostra

\begin{tabular}{|c|c|c|c|}
\hline & & & $\%$ \\
\hline \multirow[t]{8}{*}{ Local da cirurgia } & Aparelho digestivo & 4 & 30.76 \\
\hline & Ortopédica & 2 & 15.38 \\
\hline & Cardíaca & 2 & 15.38 \\
\hline & Abdominal & 1 & 7.70 \\
\hline & Oncológica & 2 & 15.38 \\
\hline & Outras & 1 & 7.70 \\
\hline & Não informado & 1 & 7.70 \\
\hline & Total & 13 & 100 \\
\hline \multirow[t]{4}{*}{ Duração da cirurgia } & $\leq 1$ hora & 1 & 7.70 \\
\hline & $>1$ hora & 4 & 30.77 \\
\hline & Não informado & 8 & 61.53 \\
\hline & Total & 13 & 100 \\
\hline
\end{tabular}


v.11, n. 1,2015

\begin{tabular}{llccc}
\hline \multirow{2}{*}{ Tempo de internação prévia } & $\leq 1$ dia & 1 & 7.70 \\
\cline { 2 - 5 } & $>1$ dia & 2 & 15.38 \\
\cline { 2 - 4 } & Não informado & 10 & 76.92 \\
\hline \multirow{3}{*}{ Tempo de internação total } & Total & $\mathbf{1 3}$ & $\mathbf{1 0 0}$ \\
\cline { 2 - 5 } & $\leq 5$ dias & 1 & 7.70 \\
\cline { 2 - 5 } & $>5$ dias & 3 & 23.07 \\
\cline { 2 - 5 } & Não informado & 9 & 69.23 \\
\hline & Total & $\mathbf{1 3}$ & $\mathbf{1 0 0}$ \\
\hline
\end{tabular}

Ainda sobre o procedimento cirúrgico, nos artigos que trouxeram informação a respeito ao tempo de cirurgia, a maior parte excedeu o tempo de 1 hora. Em relação ao tempo de internação prévia, a maior parte da amostra não contava com este dado. Dos artigos que trouxeram esta informação, a maior parte mostrou tempo de internação prévia maior que 1 dia. Sobre o tempo de internação total, em um trabalho (7,70\%) este tempo foi de até 5 dias, em três $(23,07 \%)$ foi maior que 5 dias e em nove (69,23\%) não havia esta informação (TABELA 5).

\section{DISCUSSÃO}

Estudo nacional realizado pelo Ministério da Saúde no ano de 1999 encontrou uma taxa de infecção de sítio cirúrgico de $11 \%$ do total de procedimentos cirúrgicos analisados (BRASIL, 2013). No presente estudo, a maior parte da amostra apresentou incidência de ISC menor que 10\%, o que demonstra que a incidência de ISC no Brasil está em processo de redução.

Levanta-se, porém, outra hipótese, a de subnotificação dos casos de ISC. No Brasil, a maior parte dos hospitais limita-se à vigilância de ocorrência de ISC no período de internação e não inclui 
o acompanhamento sistemático dos pacientes cirúrgicos após a alta hospitalar. Segundo Batista \& Rodrigues (2012) de 12 a 84\% das ISC são diagnosticadas durante a vigilância pós-alta hospitalar, por isso, visando reduzir a subnotificação dessas infecções é necessário indicadores acurados para reconhecimento fidedigno da ISC. Estratégias como o acompanhamento direto do paciente no retorno ambulatorial, na retirada de pontos e na vigilância dos casos de reinternação ajudam na detecção desses casos.

No que se refere aos microorganismos mais prevalentes, a amostra revelou - se concordante com a literatura já que, segundo o manual da Organização Mundial de Saúde - OMS “Cirurgias Seguras Salvam Vidas”, os patógenos responsáveis pelas infecções de sítio cirúrgico variam de acordo com o tipo de cirurgia, com o órgão e a localização, e o patógeno mais frequentemente isolado é o Staphylococcus aureus (OMS, 2009).

Tendo em vista que a microbiota exógena é composta primariamente por microorganismos aeróbios, especialmente pelas bactérias Gram positivas (como estafilococos e estreptococos). Infere-se que a contaminação da ferida operatória por Staphylococcus aureus pode se dar através dos instrumentos cirúrgicos, da aparelhagem e móveis da sala operatória, de próteses e outros implantes, do contato da equipe de cirurgia com o sítio cirúrgico; ou seja, grande parte das ISC poderiam ser evitadas através de intervenções mínimas como a correta desinfecção e esterilização de artigos utilizados nas cirurgias e da própria sala de cirurgia, bem como a correta paramentação da equipe e antissepsia correta da pele (OMS, 2009).

O controle e a prevenção da ISC começam com o preparo do paciente em relação a pele, tricotomia, roupa privativa, retirada de adornos, preparo da equipe cirúrgica em relação a unhas, roupa privativa e as paramentações cirúrgicas, cuidado do ambiente com limpeza de sala operatória, piso, padrões de circulação e procedimentos com assepsia, escovação cirúrgica, colocação de campos esterilizados, validade da esterilização e manuseio do material estéril, funções das quais a equipe de enfermagem é responsável (KUNZLE, 2006).

Sobre os fatores relacionados ao paciente, os dados obtidos nesta e em outras pesquisas são pequenos e não permitem afirmar com clareza se o sexo é um fator de risco; quanto à idade, na literatura ainda não há um consenso, mas sabe-se que os extremos de idade podem favorecer o desenvolvimento da ISC; para Batista \& Rodrigues, (2012) pacientes nos extremos de idade - 


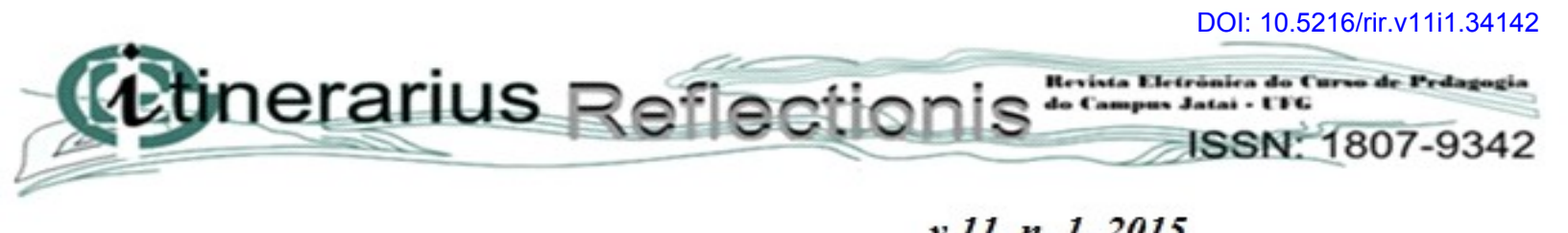

$$
\text { v.11, n. } 1,2015
$$

menores de um ano e maiores de 60 anos -, particularmente, pertencem ao grupo sob maior risco de ISC. O resultado no presente estudo mostra-se concordante com a afirmação, pois a maior parte dos casos de ISC aconteceu em indivíduos com mais de 50 anos de idade.

Nos aspectos que dizem respeito ao procedimento cirúrgico, a amostra demonstra que as cirurgias onde mais casos de ISC houveram foram as contaminadas e potencialmente contaminadas e que o tempo prolongado de cirurgia aumentou a taxa de ISC. Tempo intraoperatório prolongado aumenta o risco de contaminação, aumenta a lesão tecidual, aumenta a imunossupressão por perda de sangue, diminui o efeito do antibiótico profilático, aumenta o número de suturas e o uso de cautérios (AVILA, 2010).

Ainda sobre os fatores de risco relacionados ao ato cirúrgico, a amostra sugere que a redução do tempo de internação pode ser um fator positivo na prevenção da ISC, pois quanto maior o tempo de internação, mais casos de ISC houveram. O manual da OMS, 2009 "Cirurgias Seguras Salvam Vidas" traz a informação de que o tempo de internação pré-operatório prolongado bem como a permanência pós-operatória prolongada no hospital tem sido frequentemente associados ao aumento do risco de infecção de sitio cirúrgico. Além disso, ainda segundo o manual, minimizar o tempo de cirurgia é considerado como um dos principais métodos de prevenção de ISC (OMS, 2009).

Em estudo realizado por Ribeiro et al, (2013) a variável tempo total de internação apresentou relação estatisticamente significativa com presença ou não de infecção, ou seja, a ocorrência de ISC foi mais frequente nos pacientes que permaneceram internados por mais tempo; além disso, os autores também sugerem que o período longo de internação eleva os custos hospitalares, tanto com a estadia e tratamento do paciente, quanto com o diagnóstico e complicações. 


\section{CONSIDERAÇÕES FINAIS}

O patógeno mais frequentemente relacionado à ISC é o Staphyloccocus aureus; que os portadores são, em sua maioria, homens com mais de 50 anos de idade; que as cirurgias mais susceptíveis à infecção são as contaminadas e potencialmente contaminadas; o tempo prolongado de cirurgia aumenta o risco de ISC e que a redução do tempo de internação pode ser um fator positivo na prevenção da ISC, pois quanto maior o tempo de internação, maiores as chances de ISC; confirmando o que já foi dito por outros autores.

Durante a confecção do presente estudo, outra questão pôde ser levantada: Se os fatores de risco são conhecidos e as formas de prevenção também, por que ainda há casos de ISC? Sugerindo a realização de novos estudos neste sentido.

A atuação da equipe de enfermagem, nesse cenário, é muito importante, por ser a classe profissional que geralmente acompanha o paciente em todo o período perioperatório, que é responsável pela correta higienização da sala de cirurgia, pela central de material e esterilização e pelo serviço de vigilância epidemiológica e comissão de controle de infecções relacionadas à assistência de saúde - CCIRAS.

Infere-se, portanto, que grande parte das infecções de sítio cirúrgico podem ser evitadas através de intervenções mínimas; que a vigilância e empenho multidisciplinar é um ponto importante, que quanto mais conhecidos, estudados e divulgados os fatores de risco e de proteção, maiores as chances de redução dos índices de ISC, e que a enfermagem tem papel fundamental nesse contexto, sendo o elo entre o paciente e todos os outros profissionais envolvidos, devendo, portanto, estar sempre atenta e atualizada quanto a ISC. 


$$
\text { v.11, n. } 1,2015
$$

\section{REFERÊNCIAS}

AVILA, C. E. F.; et al. Relato de caso: Infecção de Sítio Cirúrgico após Cirurgia de Whipple. Com Ciências saúde. v. 20, n.3, p.253-60, 2010.

BATISTA, T. F.; RODRIGUES, M. C. S. Vigilância de infecção de sítio cirúrgico pós-alta hospitalar em hospital de ensino do Distrito Federal, Brasil: estudo descritivo retrospectivo no período 2005-2010. Epidemiol Serv Saúde. v. 21, n.2, p. 253-64, 2012.

BRASIL. Agência Nacional de Vigilância Sanitária. Critérios Diagnósticos de Infecção relacionada à assistência à saúde. 2013.

BURGATTI, J. C.; LACERDA, R. A. Revisão sistemática sobre aventais cirúrgicos no controle da contaminação/infecção do sítio cirúrgico. Rev Esc Enferm USP. v. 43, n.1, p. 237-44, 2009.

CARNEIRO, G. G. B.; et al. Análise bacterioscópica e microbiológica intraoperatória de pacientes submetidos a tratamento cirúrgico de escoliose idiopática do adolescente. Coluna/Columna. v.12, n.1, p. 42-4, 2013.

CDC - Guideline for Prevention of Surgical Site Infection, 1999.

CUNHA, E. R.; et al. Eficácia de três métodos de degermação das mãos utilizando gluconato de clorexidina degermante (GCH 2\%). Rev Esc Enferm USP. 2011; v. 45, n.6, p.1440-45.

ERCOLE, F. F.; CHIANCA T. C. M. Infecção de sítio cirúrgico em pacientes submetidos a artroplastias de quadril. Rev Latino-am Enfermagem. v.10, n.2, p.157-65, 2002.

KUNZLE, S. R. M.; et al. Auxiliares e Técnicos de Enfermagem e controle de infecção hospitalar em centro cirúrgico: mitos e verdades. Rev Esc Enferm USP. v. 40, n. 2, p. 214-20, 2006.

MARTINS, M. A.; et al. Vigilância pós-alta das infecções de sítio cirúrgico em crianças e adolescentes em um hospital universitário de Belo Horizonte, Minas Gerais, Brasil. Cad Saúde Pública. v. 24, n.5, p.1033-41, 2008. 


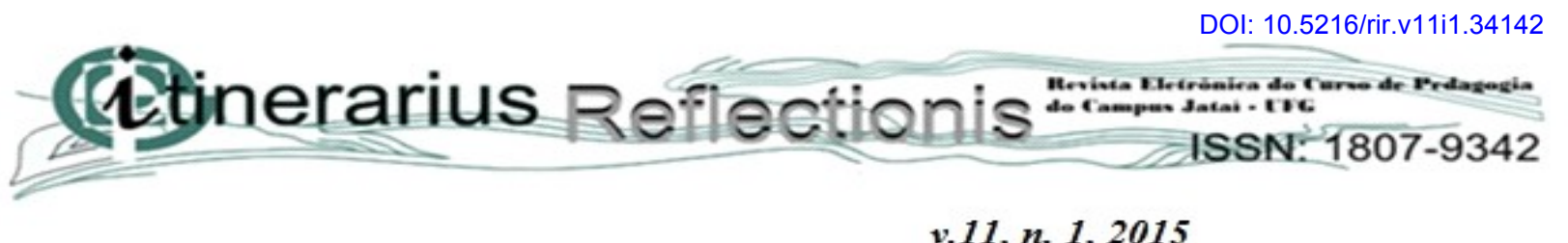

$$
\text { v.11, n. 1, } 2015
$$

MENDES, K. D. S.; SILVEIRA, R. C. C. P.; GALVÃO, C. M. Revisão integrativa: método de pesquisa para a incorporação de evidências na saúde e na enfermagem. Texto \& Contexto Enferm. v. 17, n.4, p.758-64, 2008.

OLIVEIRA, A. C.; CIOSAK, S. I. Infecção de sítio cirúrgico em hospital universitário: vigilância pós-alta e fatores de risco. Rev Esc Enferm USP. v. 41. n. 2, p. 258-63, 2007.

OLIVEIRA, A. C.; et al. Estudo comparativo do diagnóstico da infecção do sítio cirúrgico durante e após a internação. Rev Saúde Pública. v.36, n. 6, p.717-22, 2002.

OMS. Segundo desafio global para a segurança do paciente: Manual - cirurgias seguras salvam vidas (orientações para cirurgia segura da OMS) / Rio de Janeiro: OPAS; Ministério da Saúde; ANVISA, 2009.

PEREIRA M. S.; et al. A infecção hospitalar e suas implicações para o cuidar da enfermagem. Texto \& Contexto Enferm. v. 14, n. 2, p.250-57, 2005.

POVEDA, V. B.; GALVÃO, C. M.; HAYASHIDA, M. Análise dos fatores de risco relacionados à incidência de infecção do sítio cirúrgico em gastrocirurgias. Rev Esc Enferm USP. v. 37, n.1, p.819, 2003.

RIBEIRO, J. C.; et al. Ocorrência e fatores de risco para infecção de sítio cirúrgico em cirurgias ortopédicas. Acta Paul Enferm. v. 26, n.4, p. 353-59, 2013.

SILVEIRA, R. C. C. P. O cuidado de enfermagem e o cateter de Hickman: a busca de evidências. 2005. dissertação [Mestrado]. 134 f. Ribeirão Preto: Universidade de São Paulo, Escola de Enfermagem de Ribeirão Preto; 2005. 